\title{
The non-perturbative BRST quartets generated by transverse gluons or quarks in Landau gauge
}

\author{
Natalia Alkofer* \\ Institut für Physik, Karl-Franzens-Universität, Universitätsplatz, 5, A-8010 Graz, Austria \\ E-mail: natalia.alkofer@edu.uni-graz.at \\ Reinhard Alkofer \\ Institut für Physik, Karl-Franzens-Universität, Universitätsplatz, 5, A-8010 Graz, Austria \\ E-mail: reinhard.alkofer@uni-graz.at
}

\begin{abstract}
The BRST quartet mechanism is briefly reviewed. A special emphasis is given to the distinction of perturbative versus non-perturbative quartets. The field contents of the non-perturbative BRST quartets generated by transverse gluons or quarks in Landau gauge are presented. Corresponding truncated Bethe-Salpeter equations for the respective first daughter and second parent states are derived. It is discussed in which sense these equations provide evidence for the existence of bound states as daughter states in non-perturbative BRST quartets. It is noted that within the scaling solution of functional approaches the infrared divergence of the quark-gluon vertex is exactly the right one to make the respective Bethe-Salpeter equation infrared consistent.
\end{abstract}

The many faces of $Q C D$

November 2-5, 2010

Gent Belgium

\footnotetext{
*Speaker.
} 


\section{Motivation}

The gluon propagator of Landau gauge QCD has been shown to be positivity violating, see e.g. Ref. [1] and references therein. This especially implies that the one-gluon-state (treated as a physical state in perturbation theory) belongs to the states of negative norm in the indefinitemetric state space of Yang-Mills (YM) theory. As such it can be identified with a parent state in a BRST quartet whose other members, however, have to be non-perturbative, i.e. bound, states. In the following we will identify possible members of this quartet and describe a strategy to provide evidence for their role in the formalism of covariantly gauge-fixed YM theory. If successful this may provide a detailed picture of the kinematical aspects of gluon confinement in the Landau gauge. For the quark propagator the situation is less clear. Nevertheless, by following the same strategy we want to contribute to a clarification whether quarks are also positivity violating.

\section{The perturbative BRST quartet mechanism}

The perturbative BRST quartet mechanism is the generalization of the Gupta-Bleuler mechanism $[2,3]$ to YM theories, for a concise modern treatment see also $[4,5]$. The underlying idea is that the gauge condition

$$
\partial^{\mu} A_{\mu}=0
$$

as formulated in classical physics cannot be elevated consistently to an operator condition in Quantum Field Theory. The correct treatment is instead to define within the space of all quantum states of QED a physical subspace which is then given by the kernel of the operator $\partial^{\mu} A_{\mu}^{(+)}$constructed from $\partial^{\mu} A_{\mu}$ by projection on positive energies. To be concise: The physical state space contains all states $|\Psi\rangle$ which fulfill

$$
\partial^{\mu} A_{\mu}^{(+)}|\Psi\rangle=0
$$

These physical states contain then the longitudinal and the time-like photons such that their respective contributions precisely cancel. Therefore there is no contribution of unphysical states in the $S$-matrix. Due to the Minkowski metric it is unavoidable that in covariant gauges the time-like photon states are negative-metric states, and the total state space is an indefinite-metric state space.

Why then keeping the time-like and the longitudinal photon in the formalism if they cancel in all physical states? If one includes quantum fluctuations we need a tool to count them correctly. E.g. in perturbation theory in non-relativistic quantum mechanics one injects a complete set of states (i.e. a "one") to obtain the correct formulae. The analogue in relativistic quantum field theory are loops in Feynman diagrams: They describe the quantum fluctuations, and in order to count correctly one has to inject again a complete set of states, or phrased otherwise, one has to sum over the propagators of all fields in the formalism, even the unphysical ones. This way of counting is illustrated in Fig. 1.

The gauge fields of YM theories (called generically gluons in the following although the formalism, of course, is valid for all YM theories and not only the Strong Interactions) are selfinteracting. Especially the fact that transverse gluons may scatter into longitudinal and time-like ones does not allow a straightforward generalization of the Gupta-Bleuler mechanism. However, on a purely perturbative level the cancellation mechanism is only slightly more complicated: Instead 

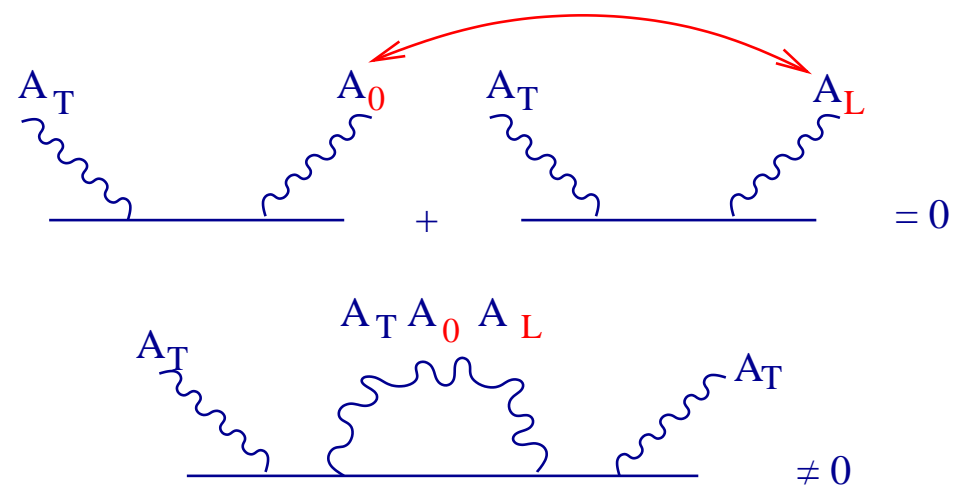

Figure 1: An illustration of the Gupta-Bleuler mechanism in covariantly gauge fixed QED.

of two respective states four do cancel against each other. In order to describe this so-called quartet cancellation mechanism we discuss first the elementary BRST quartet [6]. Within the FaddeevPopov quantization of QED one can (by simply ignoring the fact that the ghosts decouple from the gauge bosons) also formulate the elementary BRST quartet [5]. This results in, of course, the cancellation of time-like and longitudinal photons as in the Gupta-Bleuler mechanism. In non-Abelian gauge theories the elementary BRST quartet takes care of the cancellation of longitudinal and timelike gluons as well as ghosts and antighosts in all physical states. Here two remarks are in order: First, the elementary BRST quartet is also valid in the limit of gauge coupling $g \rightarrow 0$. Therefore the perturbative BRST quartet mechanism in YM is only an $m$-fold duplication of the single cancellation mechanism in QED [5] with $m$ being the dimension of the adjoint representation of the gauge group. Second, due to the nature of the BRST transform one does not directly consider the longitudinal and time-like gluons but linear superpositions of them, the forward, resp., backward polarized gluons, see e.g. Chapter 16 of Ref. [4] for a definition of these states.

The reason for building quartets is related to the nilpotency of the BRST transformation: Every non-singlet state can then produce only one further state when the BRST charge operator is applied, making thus a doublet. It proves useful to form quartets. This is done such that the Faddeev-Popov charge conjugated state of the daughter state in this doublet is used as a 2nd parent state which under BRST generates the 2nd daughter and thus completes the quartet. The construction mechanism is illustrated in Fig. 2, and we will return to it several times in the following.

To highlight the nilpotency of the BRST transformation we will work in a representation with Nakanishi-Lautrup field $B^{a}$ which becomes on-shell identical to the gauge fixing condition, $B^{a}=$ $(1 / \xi) \partial_{\mu} A_{\mu}^{a}$ where $\xi$ is the gauge parameter of linear covariant gauges. To memorize the BRST transformation $\delta_{B}$ one may picture it as a kind of gauge transformation with a constant ghost field as parameter:

$$
\begin{array}{lll}
\delta_{B} A_{\mu}^{a}=\widetilde{Z}_{3} D_{\mu}^{a b} c^{b} \lambda, & \delta_{B} q=-i g t^{a} \widetilde{Z}_{1} c^{a} q \lambda, \\
\delta_{B} c^{a}=-\frac{g}{2} f^{a b c} \widetilde{Z}_{1} c^{b} c^{c} \lambda, & \delta_{B} \bar{c}^{a}=B^{a} \lambda, & \delta_{B} B^{a}=0,
\end{array}
$$

where $D_{\mu}^{a b}$ is the covariant derivative. The parameter $\lambda$ lives in the Grassmann algebra of the ghost fields $c^{a}$ and carries ghost number $N_{\mathrm{FP}}=-1 . \widetilde{Z}_{1}$ and $\widetilde{Z}_{3}$ are the ghost-gluon-vertex and the ghost wave function renormalization constants. It has been shown that in Landau gauge $\widetilde{Z}_{1}=1$ [7]. 


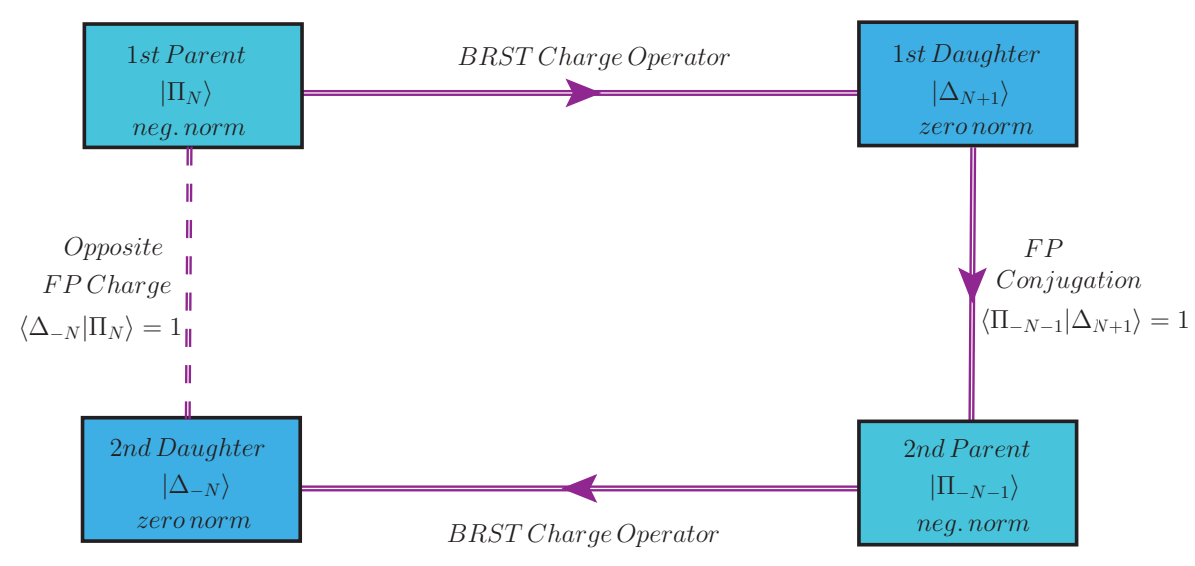

Figure 2: An illustration of the construction of a BRST quartet.

In a next step one follows the construction of Noether's theorem to derive a BRST charge operator $Q_{B}$. It generates a ghost number graded algebra on the fields, $\delta_{B} \Phi=\left\{i Q_{B}, \Phi\right\}$. Defining the ghost number operator $Q_{c}$ one obtains the algebra

$$
Q_{B}^{2}=0, \quad\left[i Q_{c}, Q_{B}\right]=Q_{B}, \quad\left[Q_{c}, Q_{c}\right]=0 .
$$

It is complete in the full and therefore indefinite metric state space of a YM theory. The BRST cohomology is then constructed as follows: The semi-definite physical subspace $\operatorname{Ker} Q_{B}$ is defined on the basis of this algebra by those states which are annihilated by the BRST charge $Q_{B}, Q_{B}|\psi\rangle=$ 0 . Since $Q_{B}^{2}=0$, this subspace contains the space $\operatorname{Im} Q_{B}$ of the so-called daughter states $Q_{B}|\phi\rangle$ (cf. Fig. 2), which are images of their parent states in the indefinite metric state space. A physical (i.e. positive-metric) Hilbert space is then obtained as the quotient space of equivalence classes:

$$
\mathscr{H}\left(Q_{B}\right)=\operatorname{Ker} Q_{B} / \operatorname{Im} Q_{B} .
$$

This Hilbert space is nothing else than the space of BRST singlets. All states are either BRST singlets or belong to quartets, this exhausts all possibilities [6]. Here a remark is in order: Had we required only $Q_{B}|\psi\rangle=0$ half of these metric partners had been eliminated from all $S$-matrix elements, leaving the unpaired daughter states of zero norm which do not contribute to any observable, $c f$. Fig. 2. However, from a mathematical point of view it is more satisfactory to retain only positive-norm states in the physical state space. Note furthermore that the parent-daughter states of opposite Faddeev-Popov charge possess non-vanishing matrix elements (which are usually normalized to one) [6]. This elucidates why quartets and not doublets are considered: These non-vanishing matrix elements are essential in the cancellation mechanism.

As BRST is a symmetry and the BRST charge operator $Q_{B}$ commutes with the Hamiltonian the daughter state is degenerate with the parent state: ${ }^{1}$

$$
H|\psi\rangle=E|\psi\rangle \Rightarrow H Q_{B}|\psi\rangle=Q_{B} H|\psi\rangle=E Q_{B}|\psi\rangle
$$

And as the Landau gauge Hamiltonian $H$ is ghost-antighost symmetric all members of a BRST quartet are degenerate.

\footnotetext{
${ }^{1}$ We thank Dan Zwanziger for pointing this out to us.
} 
The elementary quartet consists of the asymptotic states related to the backward and forward polarized gluons as well as the ghost and the antighost $[8,6]$. Hereby one gluon polarization and the antighost provide the parent states, the orthogonal gluon polarization and the ghost the daughter states. In all physical states the contribution of this quartet cancels strictly due to the algebra (2.4), a detailed description is given in Sect. 4.1 of [6]. The corresponding construction of perturbative "multi-particle" BRST quartets follows straightforwardly and is illustrated in Fig. 2. To fix the notation: We will call the negative norm state of Faddeev-Popov charge $N$ we start with the 1st parent $\Pi_{N}$. Acting with the BRST charge operator $Q_{B}$ one obtains the 1st daughter. The FaddeevPopov charge reflected state of the 1 st daughter provides the 2 nd parent. Acting on it with $Q_{B}$ provides the 2nd daughter with again has then Faddeev-Popov charge $N$.

\section{The non-perturbative BRST quartet mechanism}

Within perturbation theory the transverse gluons belong to the Hilbert space defined by the BRST cohomology. However, this is in open conflict with the observed confinement of gluons. Therefore it has been conjectured already in the seventies that the transverse gluons are also part of a BRST quartet [8]. This property is then in turn believed to be an important aspect of gluon confinement [9]. Somewhat later it has been observed [10] that the antiscreening of gluons (which is a very welcome property as it explains asymptotic freedom) is already at the perturbative level in conflict with the positivity of the gluon spectral function. As stated above there is no doubt any more that the transverse gluons of Landau gauge QCD are positivity violating, see e.g. Ref. [1].

An inspection of Fig. 2 implies that "one-transverse-gluon" states are BRST parent states. Their respective daughters, however, cannot be the elementary "one-ghost" states because these are members of the elementary quartet. From eq. (2.3) it immediately follows that the 1st daughter state of an "one-transverse-gluon" state needs to have the field content $\widetilde{Z}_{3} f^{a b c} A_{\mu}^{c} c^{b}$. For every "onetransverse-gluon" state there should occur exactly one degenerate daughter state. This implies the existence of a ghost-gluon bound state in the adjoint representation [11]. In this sense the resulting BRST quartet is strictly non-perturbative because the formation of bound states cannot be described with perturbation theory. The Faddev-Popov charge reflected 2nd parent state is then an antighostgluon bound state. In this context Landau gauge provides an advantage as compared to general linear covariant gauges: In the limit $\xi \rightarrow 0$ the formalism becomes ghost-antighost-symmetric, and thus the existence of a ghost-gluon bound state implies the occurrence of a degenerate antighostgluon bound state with same quantum numbers. Even having then the 2nd parent, the BRST transformation (2.3) leaves then three possibilities for the 2nd daughter: a ghost-antighost bound state, a ghost-antighost-gluon bound state, or a bound state of two differently polarized gluons.

Besides the almost trivial observation that, if a BRST quartet is generated by quarks it can only be a non-perturbative one, containing a ghost-quark bound state as 1st daughter not much is known about BRST quartets generated by quarks. It is also unknown whether quarks violate positivity. Although for light quarks dynamical chiral symmetry breaking (and for heavy quarks explicit chiral symmetry breaking) determines the infrared behaviour of the quark propagator the analytic structure of the quark propagator is highly sensitive to details in the quark-gluon vertex, see, e.g., Ref. [12]. The quark-gluon vertex for light quarks is, on the other hand, also very strongly influenced by dynamical chiral symmetry breaking $[13,14,15]$. The mass generation for quarks 
related to chiral symmetry breaking depends strongly on details of the dynamics. Which mechanism then guarantees that the corresponding bound states are degenerate with the quark states is completely unknown. We therefore hope that an investigation of non-perturbative BRST quartets at least partially will help to resolve these questions.

\section{Properties of ghost-gluon bound states from infrared Landau gauge YM theory}

By now quite some information on the infrared behaviour of Landau gauge YM theory is available. Especially, in the deep infrared general properties have been deduced by employing functional equations. Dyson-Schwinger equation studies have been extended from a previous analysis of gluon and ghost propagators [16, 17, 18, 19, 20] to all Yang-Mills vertex functions [21, 22, 23]. Functional Renormalization Group Equations allow a further restriction on the solution for the Green's functions: There is one unique scaling solution with power laws for the Green's functions $[24,25]$ and a one-parameter family of solutions, the so-called decoupling solutions. The latter are infrared trivial solutions which possess as an endpoint exactly the scaling solution characterized by infrared power laws. Numerical solutions of the decoupling type (there called "massive solution") have been published in [26, 27] and references therein. A recent detailed description and comparison of these two types of solutions has been given in Ref. [28], see also Refs. [29, 30, 31, 32]. Most lattice calculations of the gluon propagator favor a decoupling solution. However, in Ref. [33] it has been suggested that the infrared behaviour of the Green's function may depend on the nonperturbative completion of the gauge.

The scaling solution respects BRST symmetry whereas every decoupling solution breaks it [28], although very likely only softly. Being very strictly, the analysis as presented below will be only valid if the scaling solution is a correct one. The situation is, however, not as severe as it seems. First, if the conjecture of Ref. [33] is correct it is sufficient that only one non-perturbative completion of Landau gauge with scaling solution exists to make the analysis of Ref. [11] wellfounded. Second, even if only decoupling type of solutions were correct an extended BRST-like nilpotent symmetry is likely to take the role of the BRST symmetry [37], or the soft BRST symmetry breaking can be treated as spontaneous symmetry breaking [38], see also Ref. [39] and references therein, as well as the discussion below. It is important to realize that all arguments about infrared dominance of diagrams stay correct: The numerical value of a diagram which is infrared leading in the scaling solution will be large in a physically acceptable decoupling solution.

All one-particle irreducible Green's functions in the scaling solution in the simplified case with only one external spacelike scale $p^{2} \rightarrow 0$ obey a simple power law. For a function with $n$ external ghost and antighost as well as $m$ gluon legs one obtains:

$$
\Gamma^{n, m}\left(p^{2}\right) \sim\left(p^{2}\right)^{(n-m) \kappa} .
$$

Hereby the best known value of $\kappa$ is calculated from truncated equations and is given by $\kappa=0.595$ $[18,19]$. The above solution fulfills all functional equations and all Slavnov-Taylor identities. It verifies the hypothesis of infrared ghost dominance [34].

As already emphasized gluons violate positivity $[1,12]$. For the scaling solution this can be immediately deduced from the fact that for this solution the gluon propagator vanishes at zero virtuality, $p^{2}=0$, with an exponent $2 \kappa-1$. It leads to an infrared diverging ghost propagator 
with exponent $-\kappa-1$ as well as infrared diverging three- and four-gluon vertex functions $(-3 \kappa$ and $-4 \kappa$, respectively). A further important property of the scaling solution is the infrared trivial behaviour of the ghost-gluon vertex which is in agreement with general arguments [7, 19].

The ghost-gluon bound state is looked for in the ghost-gluon scattering kernel. To this end we want to truncate this quantity to the infrared leading term. We use the MATHEMATICA package DoDSE $[35,36]$ to derive the diagrammatic expressions for the Dyson-Schwinger equation of this four-point function. A diagram-by-diagram infrared power counting is performed by attributing anomalous infrared exponents to the internal legs and vertex functions. A ghost propagator provides a $-\kappa$, a gluon propagator a $2 \kappa$, the vertex functions the powers cited above. It is somewhat lengthy but straightforward to verify that in the scaling solution the infrared exponent of the ghost-gluon scattering kernel is $-\kappa$. More important, the infrared power counting also provides the infrared leading terms.

With two different fields involved there are two distinct possibilities for the Dyson-Schwinger equation according to which leg one puts the bare vertex. Placing the bare vertex to a ghost leg provides a consistent infrared counting [11].
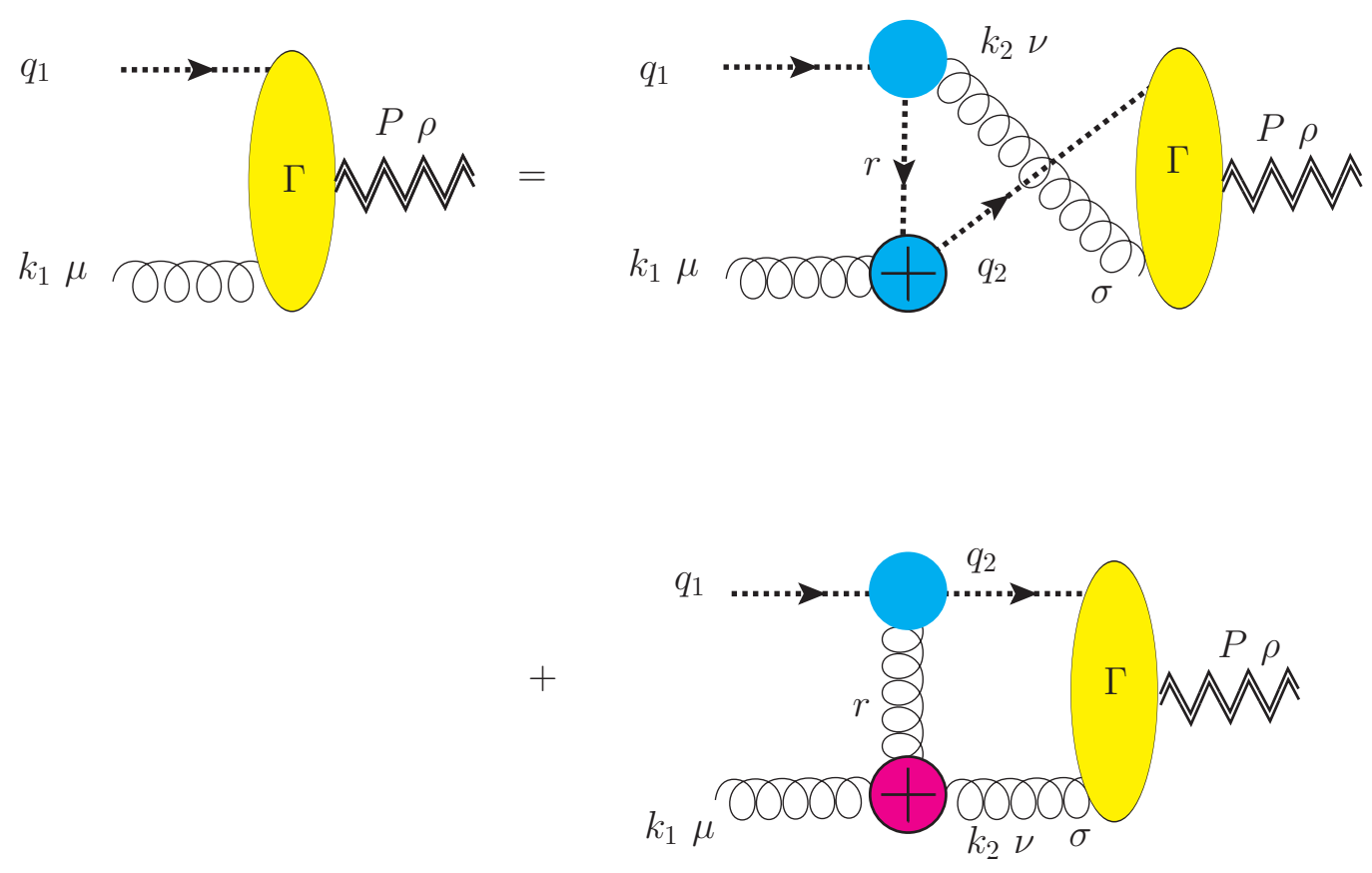

Figure 3: Graphical representation of the gluon-ghost Bethe-Salpeter equation. Crosses denote dressed vertices.

The truncation process for the diagrams on the r.h.s to be kept is: It should contain the oneparticle irreducible ghost-ghost-gluon-gluon four-point function and no $n \geq 5$-point function, it should be infrared leading, and the interaction shall take place in the ghost-gluon channel. This leaves two diagrams: One with two ghost and one gluon propagator on internal lines. This is effectively a ghost exchange. And another one with two gluon and one ghost propagator on internal lines. This is a gluon exchange. Note that this diagram is infrared leading because in the scaling solution the fully dressed three-gluon vertex is infrared divergent. 
Assuming the existence of a bound state as well as employing the usual decomposition of the (ghost-ghost-gluon-gluon) four-point function into Bethe-Salpeter amplitudes and performing the expansion around the pole (see e.g. Sect. 6.1 of Ref. [40]) one arrives at the Bethe-Salpeter equation depicted in Fig. 3. Using the propagator parameterizations of e.g. Ref. [12], the ghost-gluon vertex of Ref. [41], and the three-gluon vertex of Ref. [42] one can derive a self-consistent equation for the corresponding Bethe-Salpeter amplitude containing otherwise only known quantities [11]. The decisive property of the kernels of this Bethe-Salpeter equation are: For the upper diagram of the r.h.s of Fig. 3 the kernel is well represented by

$$
\alpha^{\mathrm{g} h}\left(r^{2}\right) / r^{2} \quad \text { with } \quad \alpha^{\mathrm{g} h}\left(r^{2}\right)=\frac{g^{2}}{4 \pi} G^{2}\left(r^{2}\right) Z\left(r^{2}\right) .
$$

( $G$ and $Z$ are the ghost and gluon renormalization function, respectively.) For the lower diagram the corresponding expression is $\sqrt{\alpha^{\mathrm{g} h}\left(r^{2}\right)} \sqrt{\alpha^{3 g}\left(r^{2}\right)} / r^{2}$ with $\alpha^{3 g}\left(r^{2}\right)$ being proportional to the square of the three-gluon vertex and $Z^{3}$. As the coupling constant derived from the 3 -gluon vertex has a smaller infrared fixed point [42] than the one derived from the ghost-gluon vertex the upper diagram will be dominant. With $\alpha^{\mathrm{gh}}(0)=8.92 / N_{c}$ (see e.g. [43] or Sect. 2.3 of Ref. [44]) it is evident that the kernel of the ghost-gluon Bethe-Salpeter equation is very strong. As typical strengths for critical coupling constants are of the order of one (see e.g. Ref. [40]) one may even speculate whether the kernel of this Bethe-Salpeter equation provides evidence for a dynamical breaking of BRST symmetry. A very welcome side effect would be the related Goldstone nature of the bound state guaranteeing masslessness.

\section{On the quark-gluon bound state equation}

The scaling solution for the YM Green's functions leads to dynamical chiral symmetry breaking in the quark sector [15]. The quark propagator is then infrared finite. The twelve possible Dirac tensor structures of the quark-gluon vertex are then all infrared divergent with an infrared exponent $-\kappa-1 / 2$. The same infrared divergence results for vanishing gluon momentum, and this leads to an $1 / k^{4}$ behaviour of the kernel in the four-quark function, $k$ being the momentum exchange. This is indicative of a linearly rising potential between static quarks, and thus quark confinement. Furthermore, the Slavnov-Taylor identities require that the ghost-ghost-quark-quark scattering kernel is infrared trivial, see Sect. 3.9 in Ref. [15].
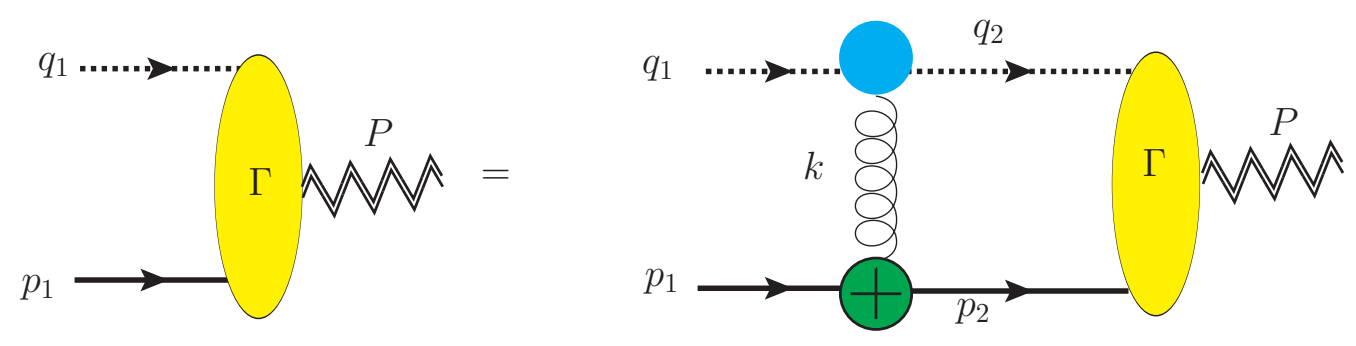

Figure 4: Graphical representation of the quark-ghost Bethe-Salpeter equation.

As in the ghost-gluon case one has two choices for the Dyson-Schwinger equation for the quark-ghost scattering kernel according to which leg one puts the bare vertex. Choosing a ghost 
leg to place the bare vertex is the infrared consistent choice [11]. Using the same truncation requirements and the same derivation of the Bethe-Salpeter equation as in the previous subsection one arrives at the equation depicted in Fig. 4. This equation is in full agreement with the infrared analysis of the scaling solution, i.e. it is a valid bound state equation, and in its kernel the infrared exponent $\kappa$ cancels.

Furthermore, this kernel is well approximated by $\sqrt{\alpha^{\mathrm{g} h}\left(k^{2}\right)} \sqrt{\alpha^{\mathrm{q}-g l}\left(k^{2}\right)} / k^{2}$ where $\alpha^{\mathrm{g} h}\left(k^{2}\right)$ is defined above and $\alpha^{\mathrm{q}-g l}\left(k^{2}\right)$ is proportional to the square of the quark-gluon vertex and $Z$. As $\alpha^{q-g l}\left(k^{2}\right) \propto 1 / k^{2}$ the above remarks of the super-criticality of the kernel equally apply.

\section{Conclusions and outlook}

In these notes we briefly reviewed the concept of BRST quartets, and we emphasized the different roles of the perturbative and non-perturbative BRST quartets. We have discussed a possibility how the non-perturbative BRST quartets generated by transverse gluons and quarks can be studied quantitatively.

To complete this project many open questions still needs to be answered: What are the bound states representing the respective 2nd daughters? Is BRST spontaneously broken? Are there associated Goldstone bosons or fermions? Can a solution of the homogeneous or inhomogeneous Bethe-Salpeter equation provide information on the positivity or positivity violation for quarks? And what is then the relation to quark confinement?

\section{Acknowledgments}

We thank the organizers of this workshop for all their efforts which made this highly interesting workshop possible. We are grateful to many of the participants for interesting discussions.

\section{References}

[1] P. O. Bowman et al., Phys. Rev. D 76 (2007) 094505 [arXiv:hep-lat/0703022].

[2] S. N. Gupta, Proc. Phys. Soc. A 63 (1950) 681.

[3] K. Bleuler, Helv. Phys. Acta 23 (1950) 567.

[4] M. E. Peskin and D. V. Schroeder, "An Introduction To Quantum Field Theory," Reading, USA: Addison-Wesley (1995) $842 p$.

[5] S. Weinberg, "The quantum theory of fields. Vol. 2: Modern applications," Cambridge, UK: Univ. Pr. (1996) $489 p$.

[6] N. Nakanishi and I. Ojima, World Sci. Lect. Notes Phys. 27 (1990) 1.

[7] J. C. Taylor, Nucl. Phys. B33 (1971) 436.

[8] T. Kugo and I. Ojima, Prog. Theor. Phys. Suppl. 66, 1 (1979).

[9] L. von Smekal and R. Alkofer, arXiv:hep-ph/0009219.

[10] R. Oehme and W. Zimmermann, Phys. Rev. D 21, 471 (1980).

[11] N. Alkofer and R. Alkofer, arXiv:1102.2753 [hep-th]. 
[12] R. Alkofer, W. Detmold, C. S. Fischer, P. Maris, Phys. Rev. D70 (2004) 014014 [hep-ph/0309077]; Nucl. Phys. Proc. Suppl. 141 (2005) 122 [hep-ph/0309078].

[13] J. I. Skullerud et al., JHEP 0304 (2003) 047 [arXiv:hep-ph/0303176].

[14] R. Alkofer, C. S. Fischer and F. J. Llanes-Estrada, Mod. Phys. Lett. A23 (2008) 1105 [arXiv:hep-ph/ 0607293].

[15] R. Alkofer et al., Annals Phys. 324 (2009) 106 [arXiv: 0804.3042 [hep-ph]].

[16] L. von Smekal, R. Alkofer and A. Hauck, Phys. Rev. Lett. 79 (1997) 3591 [arXiv:hep-ph/9705242].

[17] P. Watson and R. Alkofer, Phys. Rev. Lett. 86 (2001) 5239 [arXiv:hep-ph/0102332].

[18] D. Zwanziger, Phys. Rev. D 65 (2002) 094039 [arXiv:hep-th/0109224].

[19] C. Lerche and L. von Smekal, Phys. Rev. D 65 (2002) 125006 [arXiv:hep-ph/0202194].

[20] C. S. Fischer and R. Alkofer, Phys. Lett. B 536 (2002) 177 [arXiv:hep-ph/0202202].

[21] R. Alkofer, C. S. Fischer and F. J. Llanes-Estrada, Phys. Lett. B 611 (2005) 279 [arXiv:hep-th/0412330].

[22] M. Q. Huber et al., Phys. Lett. B 659 (2008) 434 [arXiv:0705.3809 [hep-ph]].

[23] R. Alkofer et al., Proceedings of the conference Confinement 9 [arXiv:1011.5831 [hep-ph]].

[24] C. S. Fischer and J. M. Pawlowski, Phys. Rev. D 75 (2007) 025012 [arXiv:hep-th/0609009].

[25] C. S. Fischer and J. M. Pawlowski, Phys. Rev. D 80 (2009) 025023 [arXiv:0903.2193 [hep-th]].

[26] A.C. Aguilar, D. Binosi, J. Papavassiliou, Phys. Rev. D78 (2008) 025010 [arXiv:0802.1870 [hep-ph]].

[27] P. Boucaud et al., JHEP 0806 (2008) 099 [arXiv: 0803.2161 [hep-ph]].

[28] C.S. Fischer, A. Maas and J.M. Pawlowski, Ann. Phys. 324 (2009) 2408 [arXiv:0810.1987 [hep-ph]].

[29] R. Alkofer, M. Q. Huber, K. Schwenzer, Phys. Rev. D 81 (2010) 105010 [arXiv:0801.2762 [hep-th]].

[30] M. Q. Huber, K. Schwenzer and R. Alkofer, Eur. Phys. J. C68 (2010) 581 [arXiv:0904.1873 [hep-th]].

[31] A. P. Szczepaniak and E. S. Swanson, Phys. Rev. D 65 (2002) 025012 [arXiv:hep-ph/0107078].

[32] D. Epple et al., Phys. Rev. D 77 (2008) 085007 [arXiv:0712.3694 [hep-th]].

[33] A. Maas, Phys. Lett. B 689 (2010) 107 [arXiv:0907. 5185 [hep-lat]].

[34] D. Zwanziger, Phys. Rev. D 69 (2004) 016002 [arXiv: hep-ph/0303028].

[35] R. Alkofer, M.Q. Huber and K. Schwenzer, Comput. Phys. Commun. 180 (2009) 965 [arXiv:0808.2939 [hep-th]].

[36] M. Q. Huber, Ph.D. Thesis, University Graz, 2010 [arXiv:1005.1775 [hep-th]].

[37] S. P. Sorella, Phys. Rev. D 80 (2009) 025013 [arXiv:0905.1010 [hep-th]].

[38] D. Zwanziger, Phys. Rev. D 81 (2010) 125027 [arXiv:1003.1080 [hep-ph]].

[39] S. P. Sorella et al., PoS (FacesQCD) (2011) 022 [arXiv:1102.0574 [hep-th]].

[40] R. Alkofer and L. von Smekal, Phys. Rept. 353 (2001) 281 [arXiv:hep-ph/0007355].

[41] W. Schleifenbaum et al., Phys. Rev. D 72 (2005) 014017 [arXiv:hep-ph/0411052].

[42] R. Alkofer, M. Q. Huber, K. Schwenzer, Eur. Phys. J. C 62 (2009) 761 [arXiv:0812.4045 [hep-ph]].

[43] R. Alkofer, C. S. Fischer and L. von Smekal, Acta Phys. Slov. 52 (2002) 191 [arXiv:hep-ph/0205125].

[44] C. S. Fischer, J. Phys. G: Nucl. Part. Phys. 32 (2006) R253 [arXiv:hep-ph/0605173]. 